\title{
Molecular Analysis of Carbapenem-Resistant Enterobacteriaceae at a South Korean Hospital
}

\author{
Miyoung Lee ${ }^{1,2}$ and Tae-Jin Choi ${ }^{1 *}$ \\ ${ }^{1}$ Department of Microbiology, Pukyoung National University, Busan 48513, Republic of Korea \\ ${ }^{2}$ Department of Laboratory Medicine, BHS Hanseo Hospital, Busan 48253, Republic of Korea
}

Received: February 17, 2020 / Revised: May 5, 2020 / Accepted: May 6, 2020

\begin{abstract}
The prevalence of carbapenem-resistant Enterobacteriaceae (CRE) is increasing globally, resulting in high mortality rates. Although CRE is a relatively recent problem in Korea (the first case was not diagnosed until 2010), it is responsible for serious morbidities at an alarming rate. In this study, we carried out a molecular genetic analysis to determine the incidence of CRE and carbapenemase-producing Enterobacteriaceae (CPE) at a general hospital in Korea between August 2017 and August 2019. Forty strains of CPE were isolated from various clinical specimens and analyzed via antimicrobial susceptibility testing, polymerase chain reaction to detect $\beta$-lactamase genes, deoxyribonucleic acid sequencing, multilocus sequence typing, curing testing, and conjugal transfer of plasmids. The results demonstrated that all 40 isolates were multidrug-resistant. The fluoroquinolone susceptibility test showed that $75 \%$ of the Enterobacteriaceae isolates were resistant to ciprofloxacin, whereas $72.5 \%$ were resistant to trimethoprim-sulfamethoxazole. Further, conjugation accounted for $57.5 \%$ of all resistant plasmid transfer events, which is 4.3 -fold higher than that observed in 2010 by Frost et al. Finally, the high detection rate of transposon Tn4401 was associated with the rapid diffusion and evolution of CPE. Our results highlight the rapid emergence of extensively drugresistant strains in Korea and emphasize the need for employing urgent control measures and protocols at the national level.
\end{abstract}

Keywords: Fluoroquinolone, blaKPC, Escherichia coli ST410, Klebsiella pneumoniae ST307, carbapenem-resistant Enterobacteriaceae, carbapenemase-producing Enterobacteriaceae

\section{Introduction}

Approximately 700,000 people die annually from antibiotic-resistant infections, and this number will expectedly surpass 10 million deaths per year by 2050 [1]. Antibiotic-resistant, gram-negative bacteria pose a serious threat worldwide, and, owing to the lack of available therapeutic options, the Centers for Disease Control and Prevention (CDC) has listed carbapenem-resistant members of the family Enterobacteriaceae as an "immediate public health threat that requires urgent and aggressive

\section{*Corresponding author}

Tel: +82-51-629-5617, Fax: +82-51-629-5619

E-mail: choitj@pknu.ac.kr

○ 2020, The Korean Society for Microbiology and Biotechnology action" [2]. Two resistant forms of Enterobacteriaceae are of particular concern in the hospital setting: carbapenem-resistant Enterobacteriaceae (CRE) and carbapenemase-producing Enterobacteriaceae (CPE). Both CRE and $\mathrm{CPE}$ are associated with high mortality owing to their resistance to all available antibiotics.

In Korea, the first person infected with carbapenemresistant Enterobacteriaceae was reported in December 2010. In 2014, a sudden increase in Klebsiella pneumoniae carbapenemase (KPC) cases was observed nationwide, initially occurring as hospital-based outbreaks [3], which subsequently led to inter-hospital or inter-regional spread [4], thereby resulting in the endemic stage of KPC in 2015. Following this outbreak, numerous cases of CRE and $\mathrm{CPE}$ were reported in 
Korea. As the mortality associated with invasive infections caused by CPE is extremely high [5], the spread of $\mathrm{CPE}$ is of immense clinical concern.

$\beta$-Lactamases are grouped into four classes according to their amino acid sequence: class A includes KPC and Temoneira $\beta$-lactamase (TEM); class B consists of metallo$\beta$-lactamases, such as Verona integron-encoded metallo- $\beta$ lactamases (VIM), Imipenem (IMP), and New Delhi metallo- $\beta$-lactamase-1(NDM-1); class C includes AmpC $\beta$ lactamases; and class D consists of oxacillinase (OXA)type enzymes. Carbapenem resistance in $K$. pneumoniae has been reported in most countries and can be attributed to plasmid-mediated AmpC cephalosporinases. AmpC cephalosporinases are associated with porin modifications $[6,7]$ and reduced outer membrane permeability owing to porin loss in combination with the production of either extended-spectrum $\beta$-lactamases (ESBLs) or $\beta$-lactamases, which are capable of hydrolyzing carbapenems (carbapenemases) [8, 9].

The dissemination of the blaKPC gene, encoding $\beta$-lactamase, occurs by the clonal spread of bacterial hosts harboring the gene or by horizontal transfer of transposons and plasmids carrying the gene [10, 11]. Plasmids are transmissible by conjugation [12-14]. Smillie et al. reported that approximately $14 \%$ of full-sequenced plasmids were predicted to be conjugative [15]. Hence, conjugation is one of the most effective mechanisms used to spread genetic elements among bacteria [16]. The highly mobile 10-kb Tn3-type transposon Tn4401 was considered the primary cause of the successful dissemination of blaKPC [17].

Carbapenems are $\beta$-lactam antibiotics employed as the last line of treatment during antibiotic therapy. There are few treatment options for patients infected with carbapenem-resistant CRE and CPE. Therefore, it is of great clinical significance to effectively slow down the rate of resistance transfer until a new antibiotic is available. Taking this into consideration, it is possible to slow down the rate of infection through active monitoring of CPE and by strengthening clinical management programs. Management in the form of a coping protocol to identify the antibiotic resistance risk, guidelines for the effective use of antibiotics, and the identification of trends in carbapenem-resistant enterobacteria are required for effective prevention. Moreover, these measures could play essential roles in understanding and controlling the spread of carbapenem-resistant, gramnegative pathogens.

In this study, we evaluated the incidence of CRE and CPE in a single general hospital in South Korea and characterized the $\beta$-lactamases in CRE clinical isolates.

\section{Materials and Methods}

\section{Patient description}

The study was performed at a general hospital in Busan, South Korea, between August 2017 and August 2019. During the study period, all non-duplicated clinical isolates of Enterobacteriaceae obtained from inpatients that exhibited a reduced sensitivity to carbapenems were characterized. This prospective study was performed in accordance with the ethical standards given in the Declaration of Helsinki and was approved by the Institutional Review Board of the BHS Hanseo Hospital, Busan, Korea (approval number CTS-19-004).

\section{Bacterial isolates and antimicrobial susceptibility testing}

Clinical isolates were identified by standard microbiological procedures and VITEK-2 (bioMérieux Vitek, USA). The identification of all isolates was confirmed by 16S ribosomal DNA (16S rDNA) sequencing [18]. Antimicrobial susceptibilities were determined using VITEK 2 AST N224 cards (bioMérieux Vitek) and disk diffusion tests on Mueller-Hinton agar (Becton Dickinson, USA) according to the Clinical and Laboratory Standards Institute (CLSI) guidelines [19]. Susceptibility testing was performed with the following antibiotics: ampicillin, aztreonam, cefotaxime, ceftazidime, cefoxitin, ciprofloxacin, amikacin, gentamicin, imipenem, meropenem, ertapenem, and colistin. The minimum inhibitory concentration (MICs) for colistin were assessed via the broth microdilution method using cation-adjusted Mueller-Hinton broth (Becton Dickinson) following the Clinical and Laboratory Standards Institute (CLSI) guidelines [19]. The susceptibility test results for tigecycline were confirmed by the E-test (bioMérieux, France).

For the modified carbapenem inactivation method test, organisms were incubated with a meropenem disk in tryptic soy broth (Difco Laboratories, USA). For the ethylene diamine tetra acetic acid (EDTA)-modified carbapenem inactivation method test, EDTA was added to the broth to chelate metal ions necessary for the metallo- 
$\beta$-lactamase function. Following incubation, the disks were fished out and placed on a lawn of susceptible Escherichia coli (ATCC 25922) to determine whether the test organisms degraded meropenem. Zone diameters were measured and interpreted following the CLSI guidelines [19].

\section{Genotyping of $\beta$-lactamases and the outer membrane protein}

The $\beta$-lactamase-encoding gene was evaluated by polymerase chain reaction (PCR). PCR was also performed to detect genes encoding carbapenemases (IMP-1-type, VIM-2-type, NDM, KPC, Guiana-extended-spectrum $\beta$ lactamase, and OXA-48-like) and ESBLs (CTX (cefotaximase) -M-1-, CTX-M-9-, TEM-, and sulfhydryl variant (SHV)-type) [20]. CPE isolates were also examined for the presence of plasmid-mediated AmpCs (blaACT, blaACC, blaCMY, and blaDHA), aminoglycoside resistance determinants (ARD, including armA, rmtA, rmtB, and $\mathrm{rmtD}$ ) [21, 22], and fluoroquinolone resistance determinants (including qepA, qnrA, qnrB, and qnrS) as previously described [23]. PCR was performed to detect the genes encoding the outer membrane protein (OmpK35, OmpK36) [24].

\section{Isotyping the Tn4401 transposon}

To determine the isotypes of $\operatorname{Tn} 4401$, isotype-specific forward primers $(\mathrm{a}-\mathrm{e})$ for the five most common isotypes [17] were newly designed (Table S1), and PCR was carried out with a universal reverse primer targeting blaKPC. The amplicons were sequenced for verification [25].

\section{Multilocus sequence typing (MLST)}

PCR and sequencing (Table S2) were carried out for seven housekeeping genes: $a d k$, fum $C$, gyrB, icd, $m d h$, purA, and recA for E. coli [26]; gapA, infB, mdh, pgi, phoE, rpoB, and tonB for K. pneumoniae [27]; and $a s p C$, $c l p X$, fadD, $m d h$, arcA, dnaG, and lysP for Citrobacter freundii. Nucleotide sequences were compared with those in the MLST database (http://bigsdb. web.pasteur.fr/ klebsiella for $K$. pneumoniae and http://mlst.warwick.ac.uk/mlst/dbs/Ecoli for $E$. coli) to identify allelic types and STs.

\section{Bacterial conjugation}

For the standard agar mating method, $40 \mathrm{CPE}$ strains were used as the donor, and sodium azide-resistant $E$. coli J53 was used as the recipient [28]. Following overnight mating at $37^{\circ} \mathrm{C}$ on brain-heart infusion (BHI) agar (MB cell, USA), transconjugants were selected on BHI addition agar with $100 \mu \mathrm{g} / \mathrm{ml}$ sodium azide and $0.5 \mu \mathrm{g} / \mathrm{ml}$ IMP. The conjugated strains were identified via PCR, $\beta$-lactamase-encoding, ESBLs, plasmid-mediated AmpCs, ARD, and sensitivity to fluoroquinolones.

\section{Curing test}

KPC-carrying plasmid curing was performed via the temperature-mediated plasmid emission method. Briefly, CRE strains were subjected to elevated temperaturemediated plasmid elimination by sequential passages in BHI broth (MB cell) at $42^{\circ} \mathrm{C}$ twice daily for 2 weeks. After 2 weeks, cultures were diluted and plated onto tryptic soy infusion (TSI; Difco Laboratories Inc.) medium to obtain single colonies. Suspected cured colonies were identified by selecting colonies and replating them onto TSI medium containing $5 \mu \mathrm{g} / \mathrm{ml}$ IMP (SigmaAldrich, USA) and a control TSI medium. Colonies that failed to grow in the presence of IMP were suspected to be cured and were further analyzed for blaKPC loss by PCR as well as antibiotic susceptibility testing [29].

\section{Results}

\section{Patient characteristics}

Carbapenemases were responsible for resistance in 40 isolates (66.7\% of all 60 ertapenem-resistant Enterobacteriaceae). The mean age of patients with CPE was 81.4 years (range, 57-92 years), and 27 patients (67.5\%) were females (Fig. 1A). The 40 isolates comprised $27 \mathrm{~K}$. pneumoniae isolates (67.5\%), $9 \mathrm{E}$. coli isolates (22.5\%), 2 C. freundii isolates (5\%), 1 Klebsiella oxytoca isolate (2.5\%), and 1 Klebsiella aerogenes isolate (2.5\%) (Fig. 1B).

\section{Antimicrobial susceptibilities}

All isolated strains showed a multidrug-resistant (MDR) phenotype. According to the antibiotic susceptibility profiles, all 40 isolates were resistant to ampicillin, amoxicillin/clavulanic acid, piperacillin/tazobactam, cefazolin, cefoxitin, cefotaxime, ceftazidime, cefepime, aztreonam, ertapenem, and IMP. The results of fluoroquinolone susceptibility testing showed that 32 isolates (80\%) were resistant to ciprofloxacin, whereas the tri- 

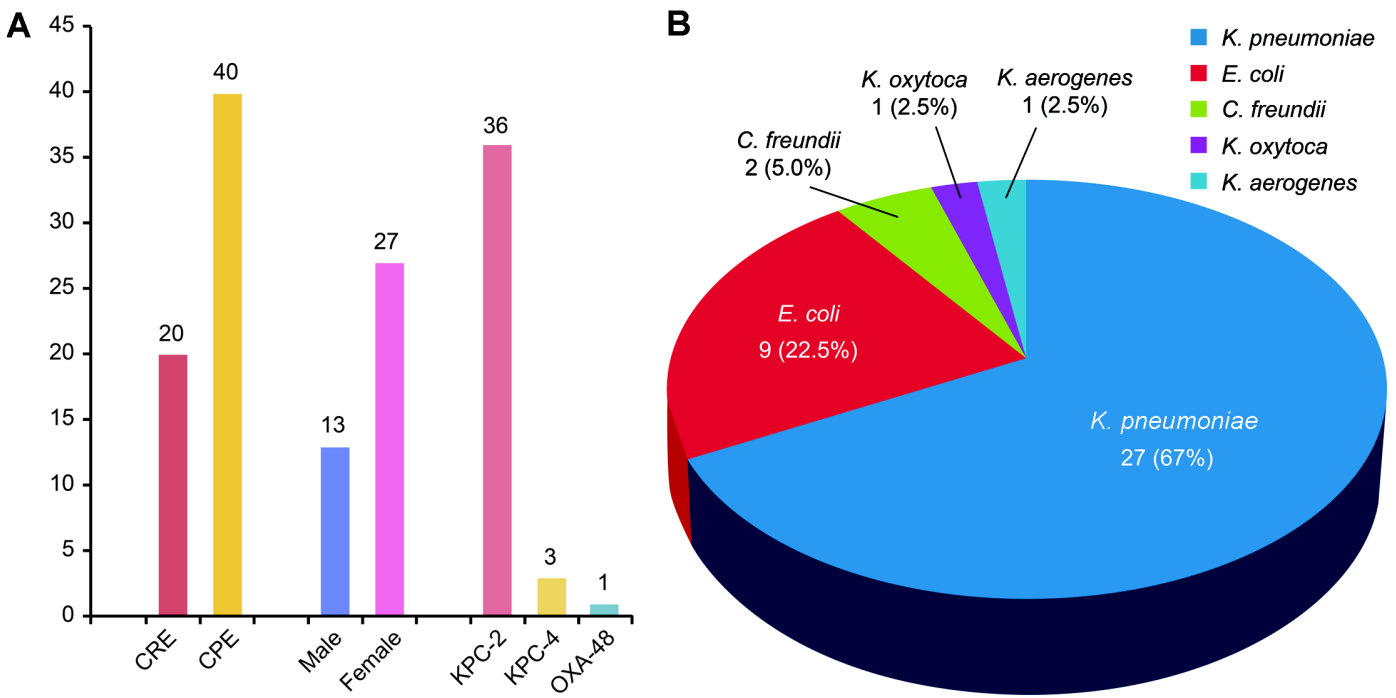

Fig. 1. Distribution of species in carbapenemase-producing Enterobacteriaceae (CPE) isolates. (A) Distribution of carbapenemase genotypes in carbapenem resistant Enterobacteriaceae isolates $(n=60)$, (B) Distribution of genotypes of CPE isolates $(n=40)$

methoprim-sulfamethoxazole resistance rates were $72.5 \%$. The aminoglycoside test results showed that 19 strains (47.5\%) were resistant to gentamicin and two strains (5\%) were intermediately resistant to amikacin. Tigecycline and colistin resistance was confirmed in two strains (5\%). The combination of $\beta$-lactamases relative to the susceptibility test results is shown in Table 1.

\section{Distribution of molecular typing}

Among the $40 \mathrm{CPE}$ isolates, 36 (90\%), 3 (7.5\%), and 1 (2.5\%) were KPC-2, KPC-4, and OXA-48 producers, respectively (Fig. 1A). We also observed a high preva- lence of ESBLs. Other $\beta$-lactamases were observed as well, including CTX-M-1 group (57.5\%), CTX-M-4 group (15\%), SHV-like (50\%), and TEM-like (32.5\%) $\beta$-lactamase isolates. Plasmid-mediated $\mathrm{AmpC}$ was rarely coproduced (4/40, 10\%); in KPC-2 producers, co-production was observed with DHA $(\mathrm{n}=2)$ and $\operatorname{ACC}(\mathrm{n}=1)$, whereas in KPC-4 producers, co-production was only observed with CIT $(n=1)$. Concerning the analysis of fluoroquinolone resistance determinants for the 40 carbapenemase strains, $67.5 \%$ of the 27 Enterobacteriaceae isolates carried a fluoroquinolone resistance determinant. The 40 carbapenemase isolates comprised 22 qnrB

Table 1. Antimicrobial susceptibilities of CPE isolates and phenotypes.

\begin{tabular}{|c|c|c|c|c|c|c|c|c|c|c|c|c|c|}
\hline \multirow{3}{*}{ CPE Isolates (\%) } & \multicolumn{10}{|c|}{ Antimicrobial susceptibility } & \multirow{2}{*}{\multicolumn{2}{|c|}{ Porin loss (\%) }} & \multirow{3}{*}{ Conjugation } \\
\hline & \multicolumn{10}{|c|}{ No. (\%) of isolates } & & & \\
\hline & FOX & FEP & ETP & IMP & AK & GN & CIP & SXT & TIG & CST & OmpK35 & OmpK36 & \\
\hline CPE strains $(n=40)$ & 0 & 0 & $1(2.5)$ & $1(2.5)$ & 39 (97.5) & $31(77.5)$ & $8(20)$ & $11(27.5)$ & $38(95)$ & $38(95)$ & $18(45)$ & $17(42.5)$ & $23(57.5)$ \\
\hline KPC-2 & 0 & 0 & 0 & 0 & $31(100)$ & $17(54.8)$ & $6(19.4)$ & $8(25.8)$ & $26(83.9)$ & $25(80.6)$ & $15(48.4)$ & $16(51.6)$ & $20(64.6)$ \\
\hline$\Delta \operatorname{Tn} 4401 a(\mathrm{n}=26)$ & 0 & 0 & 0 & 0 & $26(100)$ & $13(50)$ & $6(23.1)$ & $6(23.1)$ & $26(100)$ & $25(3.85)$ & $14(53.8)$ & $13(50)$ & $17(65.4)$ \\
\hline$\Delta \operatorname{Tn} 4401 b(\mathrm{n}=5)$ & 0 & 0 & 0 & 0 & $5(100)$ & $4(80)$ & 0 & $2(40)$ & $5(100)$ & $5(100)$ & $1(20)$ & $3(60)$ & $3(60)$ \\
\hline KPC-4 & 0 & 0 & 0 & 0 & $2(66.7)$ & $2(66.7)$ & $2(66.7)$ & $2(66.7)$ & $3(100)$ & $3(100)$ & 0 & 0 & $1(33.3)$ \\
\hline$\Delta \operatorname{Tn} 4401 b(\mathrm{n}=3)$ & 0 & 0 & 0 & 0 & $2(66.7)$ & $2(66.7)$ & $2(66.7)$ & $2(66.7)$ & $3(100)$ & $3(100)$ & 0 & 0 & $1(33.3)$ \\
\hline Total $(n=34)$ & 0 & 0 & 0 & 0 & 33 (97.1) & $19(55.9)$ & $8(23.53)$ & $10(29.42)$ & $29(85.3)$ & $28(82.4)$ & $15(44.1)$ & $16(47.1)$ & $21(61.8)$ \\
\hline $\begin{array}{l}\text { Transconjugant } \\
\text { strains }(n=23)\end{array}$ & 0 & 0 & 0 & 0 & $23(100)$ & $14(60.9)$ & $6(26.1)$ & $7(30.4)$ & $22(95.7)$ & $23(100)$ & - & - & - \\
\hline Cured strains $(n=2)$ & $1(50)$ & $1(50)$ & $2(100)$ & $1(50)$ & $2(100)$ & $2(100)$ & $1(50)$ & $1(50)$ & $2(100)$ & $2(100)$ & - & - & - \\
\hline
\end{tabular}

Abbreviations: CPE, carbapenemase-producing Enterobacteriaceae; FOX, cefoxitin; FEP, cefepime; ETP, ertapenem; IMP, imipenem; AK, amikacin; GN, gentamicin; CIP, ciprofloxacin; SXT, trimethoprim/sulfamethoxazole; TIG, tigecycline; CST, colistin. 
Table 2. Identification of resistance determinants among the 40 CPE isolates by PCR.

\begin{tabular}{|c|c|c|c|}
\hline $\begin{array}{l}\text { Antibiotic class/ } \\
\text { Resistance gene }\end{array}$ & $\begin{array}{l}\text { CPE strains } \\
\mathrm{N}=40(\%)\end{array}$ & $\begin{array}{c}\text { Transconjugants } \\
\text { strains } \\
N=23(\%)\end{array}$ & $\begin{array}{l}\text { Cured strains } \\
\mathrm{N}=2(\%)\end{array}$ \\
\hline \multicolumn{4}{|l|}{ Carbapenems } \\
\hline$K P C-2$ & $36(90)$ & $16(69.6)$ & 0 \\
\hline$K P C-4$ & $3(7.5)$ & $3(13.0)$ & 0 \\
\hline OXA-48 & $1(2.5)$ & $1(4.3)$ & 0 \\
\hline \multicolumn{4}{|l|}{ Cephalosporins } \\
\hline SHV & $20(50)$ & $11(47.8)$ & 0 \\
\hline TEM & $13(32.5)$ & $2(8.7)$ & 0 \\
\hline CTX-M-1 & $23(57.5)$ & $7(30.4)$ & $1(50)$ \\
\hline CTX-M-9 & $6(15)$ & $1(4.3)$ & 0 \\
\hline \multicolumn{4}{|l|}{$\mathrm{AmpC}$} \\
\hline$D H A$ & $2(5)$ & 0 & 0 \\
\hline$C I T$ & $1(2.5)$ & 0 & 0 \\
\hline$A C C$ & $1(2.5)$ & 0 & 0 \\
\hline \multicolumn{4}{|l|}{ Aminoglycoside } \\
\hline$r m t A$ & $1(2.5)$ & 0 & 0 \\
\hline$r m t B$ & $11(27.5)$ & $3(13.0)$ & 0 \\
\hline$r m t C$ & $2(5)$ & 0 & 0 \\
\hline$r m t D$ & $4(10)$ & 0 & 0 \\
\hline $\operatorname{armA}$ & $2(5)$ & $2(8.7)$ & 0 \\
\hline \multicolumn{4}{|l|}{ Fluoroquinolone } \\
\hline qnrA & $8(20)$ & 0 & 0 \\
\hline$q n r B$ & $17(42.5)$ & $12(52.2)$ & $2(100)$ \\
\hline qnrs & $8(20)$ & $7(30.4)$ & 0 \\
\hline qepA & $6(15)$ & $1(4.3)$ & 0 \\
\hline
\end{tabular}

Abbreviations: $\mathrm{CPE}$, carbapenemase-producing Enterobacteriaceae; KPC, Klebsiella pneumonia carbapenemase; OXA, oxacillinase; SHV, sulfhydryl variant; TEM, Temoneira $\beta$-lactamase; CTX-M, cefotaximase.

(55\%), 8 qnrA (20\%), 8 qnrS (20\%), and 6 qepA (15\%) isolates. The analysis of aminoglycoside resistance in these strains showed that 18 strains (45\%) displayed resistance: $11 \mathrm{rmtB}(27.5 \%), 4 \mathrm{rmtD}(10 \%), 2 \mathrm{rmtC}(5 \%), 2$ $\operatorname{armA}(5 \%)$, and $1 \mathrm{rmtA}(2.5 \%)$ isolates (Table 2$)$. In addition to the production of ESBLs or AmpC enzymes, the deletion or mutation of porins, such as OmpK35 and OmpK36, was reportedly correlated with increased carbapenem MICs [30]. A multi-agency survey conducted in 2013 confirmed that porins, including OmpK35 and OmpK36, were observed in 27 isolates (67.5\%) [31]. In our study, the porins OmpK35 and OmpK36 were observed in 18 (45\%) and 17 (42.5\%) isolates, respectively. OmpK35 and OmpK36 were both deleted or mutated at the same rate $(20 \%)$ (Table 3$)$.

\section{MLST}

$K$. pneumoniae was analyzed by MLST based on the analysis of the seven housekeeping genes from the 27 clinical isolates of KPC-producing $K$. pneumoniae. Using this approach, we observed five sequence types (STs). Using the $K$. pneumoniae MLST database, we detected the clones ST307 (18/27, 66.7\%) and ST11 (6/27, 22.2\%).

Furthermore, three types were identified: ST429 (1/27, 3.7\%), ST2521 (1/27, 3.7\%), and ST3660 (1/27, 3.7\%) (Table 4). E. coli MLST demonstrated that the STs ST410 and ST3520 were the most common among the carbapenemase-producing strains (2/9, 22.2\%), followed by ST117 (1/9, 11.1\%), ST617 (1/9, 11.1\%), ST720 (1/9, 11.1\%), ST457 (1/9, 11.1\%), and ST52 (1/9, 11.1\%).

\section{Isotyping the Tn4401 transposon}

The Tn4401 isotype was detected in 34 of the 40 analyzed strains (34/40, 85\%). The major isotypes associated with Tn4401 bracketing the blaKPC gene were Tn4401a (26/40, 65\%) and $\operatorname{Tn} 4401 b$ (8/40, 20\%). Tn4401b was identified in all three strains of blaKPC-4, which is consistent with the results of Jeong et al. [25]. For the six non-isotypable transposons (15\%) by the Tn4401-isotyping PCR, amplification between the $3^{\prime}$-end of the upstream ISKpn7 and the blaKPC gene was performed, and no amplicons were produced. Regarding strains carrying Tn4401, 20 strains were identified (20/34, 59\%), which increases the risk of carbapenem resistance transmission. The transposon isolates are listed in Table 4.

\section{Conjugation}

Carbapenem resistance was successfully transferred from CPE strains to $E$. coli J53 by conjugation (23/40, 57.5\%). All transconjugants exhibited significantly reduced carbapenem susceptibility, with ertapenem and imipenem MICs of 4-8 mg/l and 8-16 mg/l, respectively. In addition, the transconjugants exhibited MDR phenotypes similar to those of the clinical CPE isolate donors. The transconjugants were also resistant to cephalosporins but were susceptible to aminoglycosides (Table 1). Importantly, the transconjugant assays enabled the simultaneous transfer of blaKPC-2, blaKPC-4, blaOXA- 
Table 3. Possible genes and outbreaks identified in the CPE collection.

\begin{tabular}{|c|c|c|c|c|c|c|c|c|c|c|c|}
\hline Strain & Species & $\begin{array}{l}\text { Carbapen- } \\
\text { ems }\end{array}$ & Cephalosporins & $\mathrm{AmpC}$ & $\begin{array}{l}\text { Aminoglyco- } \\
\text { side }\end{array}$ & Fluoroquinolone & ST & Porin loss & $\begin{array}{l}\text { Tn4401 } \\
\text { variant }\end{array}$ & $\begin{array}{l}\text { Conju- } \\
\text { gants }\end{array}$ & Curing \\
\hline K1 & $K P N$ & $K P C-2$ & CTX-M-1 & & $r m t B, r m t D$ & $q n r B$ & 307 & OMPK36 & & & \\
\hline $\mathrm{K} 2$ & KPN & $K P C-2$ & $C T X-M-1$ & & $r m t B$ & $q n r B$ & 307 & OMPK36 & $b$ & + & \\
\hline K3 & $E C O$ & $K P C-2$ & TEM1, CTX-M-9 & & $\operatorname{armA}$ & & 410 & OMPK35, ОМРК36 & $a$ & + & \\
\hline K4 & KPN & $K P C-2$ & TEM1,CTX-M-1, SHV & $A C C$ & $r m t D$ & qnrB, qерA & 11 & OMPK35, ОМРК36 & b & & \\
\hline K5 & CFR & $K P C-2$ & TEM1 & $D H A$ & & $q n r A$ & 18 & & $a$ & & \\
\hline K6 & $K P N$ & $K P C-2$ & TEM1, CTX-M-1, SHV & & $r m t C$ & qnrB, qepA & 11 & OMPK36 & $a$ & + & \\
\hline K7 & $K P N$ & $K P C-2$ & TEM1, CTX-M-1, SHV & & & $q n r B$ & 11 & ОМРК35, ОМРК36 & $a$ & + & + (IMP:I) \\
\hline K8 & KPN & $K P C-2$ & TEM1, CTX-M-1, SHV & & & $q n r B$ & 11 & OMPK36 & b & + & \\
\hline K9 & KPN & $K P C-2$ & TEM1, CTX-M-1, SHV & & $r m t C$ & $q n r B, q n r S$, qepA & 307 & OMPK36 & $a$ & + & \\
\hline K10 & ECO & $K P C-2$ & $\begin{array}{l}\text { TEM1, CTX-M-1, } \\
\text { CTX-M-9, SHV }\end{array}$ & & $\operatorname{armA}$ & & 410 & & $a$ & + & \\
\hline K11 & KPN & $K P C-2$ & TEM1, CTX-M-1, SHV & & $r m t B$ & & 307 & OMPK36 & $a$ & + & \\
\hline K12 & KPN & $K P C-2$ & TEM1, CTX-M-1, CTX-M-9 & & & $q n r B$ & 307 & OMPK36 & $a$ & + & \\
\hline K13 & KOX & $K P C-4$ & & & & & & & $b$ & & \\
\hline K14 & $E C O$ & OXA48 & TEM1, CTX-M-1 & & & qnrs & 617 & & & + & \\
\hline K15 & KPN & $K P C-2$ & TEM1, CTX-M-1 & & $r m t D$ & & 11 & OMPK36 & $a$ & & \\
\hline K24 & KPN & $K P C-2$ & $C T X-M-1, S H V$ & & & & 2521 & & $a$ & + & \\
\hline K25 & KPN & $K P C-2$ & $C T X-M-1, S H V$ & & $r m t B$ & $q n r B$ & 307 & OMPK35, ОМРК36 & $a$ & + & \\
\hline K26 & $K A E$ & $K P C-2$ & SHV & & & & & OMPK36 & $a$ & + & \\
\hline K27 & $E C O$ & $K P C-2$ & SHV & & & & 720 & OMPK35 & $a$ & + & \\
\hline K28 & KPN & $K P C-2$ & SHV & & $r m t B, r m t D$ & & 307 & OMPK35, ОМРК36 & $a$ & & \\
\hline K29 & KPN & $K P C-2$ & & & $r m t B$ & qnrB, qерA & 307 & OMPK35, ОМРK36 & $a$ & & \\
\hline K102 & $K P N$ & $K P C-2$ & $S H V, C T X-M-1$ & & $r m t B$ & qnrS, qepA & 307 & OMPK35 & $a$ & + & \\
\hline K103 & $K P N$ & $K P C-2$ & $S H V, C T X-M-1$ & & $r m t B$ & qnrB, qnrS & 307 & OMPK35 & $a$ & + & \\
\hline K104 & $E C O$ & $K P C-2$ & SHV & & & & 3520 & & $a$ & & \\
\hline K105 & $E C O$ & $K P C-2$ & SHV & & & & 3520 & & $a$ & + & + \\
\hline K106 & KPN & $K P C-2$ & SHV & & & $q n r B, q n r S$ & 11 & & $a$ & & \\
\hline K107 & $E C O$ & $K P C-2$ & $S H V, C T X-M-1$ & & & $q n r A, q n r B$ & 457 & & $b$ & + & \\
\hline K108 & $K P N$ & $K P C-2$ & CTX-M-1, CTX-M-9 & & & $q n r B, q n r S$ & 307 & OMPK35 & $a$ & & \\
\hline K109 & KPN & $K P C-2$ & & & & $q n r B, q n r S$ & 307 & OMPK35 & $a$ & & \\
\hline K110 & KPN & $K P C-2$ & $S H V, C T X-M-1$ & & & $q n r B, q n r S$ & 307 & OMPK35 & $a$ & & \\
\hline K111 & $E C O$ & $K P C-2$ & & & & qnrA & 52 & & & + & \\
\hline K112 & KPN & $K P C-4$ & & & & & 3660 & & $b$ & + & \\
\hline K113 & CFR & $K P C-4$ & CTX-M-1 & CIT & & $q n r A$ & 124 & & b & & \\
\hline K114 & KPN & $K P C-2$ & TEM1 & & & $q n r A, q n r B, q e p A$ & 307 & OMPK35, ОМРК36 & $a$ & & \\
\hline K115 & KPN & $K P C-2$ & CTX-M-1 & & & $q n r A, q n r B$ & 307 & OMPK35 & & & \\
\hline K116 & KPN & $K P C-2$ & CTX-M-1 & & & $q n r A, q n r B$ & 307 & OMPK35 & & & \\
\hline K117 & $K P N$ & $K P C-2$ & $S H V, C T X-M-9$ & & $r m t B$ & $q n r A, q n r B$ & 429 & OMPK35, ОМРК36 & $a$ & + & \\
\hline K118 & ECO & $K P C-2$ & CTX-M-1 & & $r m t A$ & & 117 & & b & & \\
\hline K119 & $K P N$ & $K P C-2$ & & & $r m t B$ & $q n r B$ & 307 & OMPK35 & $a$ & + & \\
\hline K120 & $K P N$ & $K P C-2$ & & $D H A$ & $r m t B$ & $q n r B$ & 307 & OMPK35 & & + & \\
\hline
\end{tabular}

Abbreviations: CPE, carbapenemase-producing Enterobacteriaceae; KPN, Klebsiella pneumonia; ECO, Escherichia coli; CFR, Citrobacter freundii; KOX, Klebsiella oxytoca; KAE, Klebsiella aerogenes; ST, sequence type; IMP, imipenem. 
Table 4. bla $a_{\mathrm{KPC}}$ mobile elements carried by KPC producers.

\begin{tabular}{|c|c|c|c|c|c|c|c|c|c|c|c|c|c|c|}
\hline \multirow{2}{*}{$\begin{array}{c}\text { Strain } \\
\text { Sequence type }\end{array}$} & \multicolumn{5}{|c|}{ Klebsiella pneumoniae } & \multicolumn{5}{|c|}{ Escherichia coli } & \multicolumn{2}{|c|}{$\begin{array}{c}\text { Citrobacter } \\
\text { freundii }\end{array}$} & \multirow{2}{*}{$\begin{array}{c}\begin{array}{c}\text { Klebsiella } \\
\text { oxytoca }\end{array} \\
-\end{array}$} & \multirow{2}{*}{$\begin{array}{c}\text { Klebsiella } \\
\text { aerogenes } \\
-\end{array}$} \\
\hline & 307 & 11 & 429 & 2521 & 3660 & 410 & 720 & 3520 & 457 & 117 & 124 & 18 & & \\
\hline blaKPC-2 Tn4401a & $13(32.5)$ & $4(10)$ & $1(2.5)$ & $1(2.5)$ & - & $2(5)$ & $1(2.5)$ & $2(5)$ & - & - & - & $1(2.5)$ & - & $1(2.5)$ \\
\hline blaKPC-2 Tn4401b & $1(2.5)$ & $2(5)$ & - & - & - & - & - & - & $1(2.5)$ & $1(2.5)$ & - & - & - & - \\
\hline blaKPC-4 Tn4401b & - & - & - & - & $1(2.5)$ & - & - & - & - & - & $1(2.5)$ & - & $1(2.5)$ & - \\
\hline
\end{tabular}

Abbreviations: KPC, Klebsiella pneumoniae carbapenemase.

48, ESBL, and AmpC, as well as aminoglycoside and fluoroquinolone resistance-determining genes (Table 2).

\section{Curing test}

A curing test was performed to artificially remove the plasmid containing the KPC-2 gene. However, curing was successfully performed in only two strains, $\mathrm{K} 7$ and K105 (2/40, 5\%). In each of these strains, curing significantly reduced carbapenem resistance, with imipenem and ertapenem MICs of $\leq 0.5 \mathrm{mg} / \mathrm{l}$ and $0.5-2 \mathrm{mg} / \mathrm{l}$, respectively. Other antibiotics that showed similar effects on the clinical CPE isolate donors are also shown in Table 1.

\section{Discussion}

In the 1940s, the first antibiotics were derived from mold; however, since then, advances in antibiotic development has relied on chemical synthesis. However, with the emergence of multidrug resistance, the end of the antibiotic era may be near. The World Health Organization warns about a post-antibiotic era, in which no antibiotics will be able to kill bacteria because of their widespread resistance. Currently, hospitals face emerging resistance to third-generation cephalosporins and fluoroquinolones by Staphylococcus aureus and Enterococcus. Recently, there has been growing speculation about the impossibility of treating CRE in a post-antibiotic era. To the best of our knowledge, this is the first study to conduct molecular genetic analysis of CPE at a single hospital in Korea since the conduction of the CRE survey by the Korea CDC in June 2017. Here, we described the distribution of major $\beta$-lactamases in a group of extensively drug-resistant (XDR) Enterobacteriaceae isolates from a single hospital, presenting several important results.
Antibiotic resistance is increasing, and, therefore, a new classification termed "XDR" has been created. Fluoroquinolones are important therapeutics used to treat both human and animal infections [32]. The introduction of fluoroquinolones has offered clinicians the ability to treat human cases of complicated urinary tract, gastrointestinal, and respiratory tract infections as well as sexually transmitted diseases [33]. In veterinary medicine, fluoroquinolones have served as effective therapeutics for treating enteric infections and respiratory diseases in food-producing and companion animals [34]. Owing to their antimicrobial activity against a broad spectrum of pathogenic bacteria, advantageous pharmacokinetic characteristics, and low toxicity, fluoroquinolones have become attractive for use in farm animals [35, 36]. Since the late 1980s, fluoroquinolones used in human medicine have differed from those used in veterinary medicine $[37,38]$. Nonetheless, a public health concern remains, i.e. the use of fluoroquinolones in livestock may result in bacterial resistance that can be transmitted through the food chain. This can be explained by crossresistance between fluoroquinolones and enrofloxacin, a commonly used agent in farm animals, which is partially metabolized to ciprofloxacin within animals [39, 40]. Thus, resistance to antibiotics has continuously remained in the food chain and may be permanent; therefore, close management and restrictions are required in the future. Additionally, there is also an emerging resistance to colistin and tigecycline, last-resort antibiotics for CRE treatment.

Interestingly, our study showed that CRE is more susceptible to amikacin, an antibiotic of the aminoglycoside family. The EUCAST(European Committee on Antimicrobial Susceptibility Testing) guidelines state that the amikacin susceptibility status must be revised to "intermediate" should a member of the Enterobacteriaceae test 
as tobramycin intermediate or resistant, and gentamicin and amikacin susceptible [41].

Therefore, we recommend the sparingly use of amikacin to treat intestinal bacterial infections resistant to other aminoglycosides.

The most predominant KPC-producing clone reported globally is K. pneumoniae ST258 [42, 43]; however, this was not the case in South Korea. Rather, the predominant South Korean KPC producer was the K. pneumoniae ST307 clone. ST11 was also observed in Korea at a significantly lower prevalence than ST307. According to a study by Jeong et al., the four $K$. pneumoniae subclones ST307/Tn4401a[blaKPC-2], ST307/Tn4401b[bla KPC-4], ST392/Tn4401b[blaKPC-4], and ST11/ND[bla $K P C$-2] triggered inter-regional dissemination of $\mathrm{KPC}$ producers in Korea [25]. In this study, various K. pneumoniae subclones and $E$. coli subclones were isolated; the primary $K$. pneumoniae subclones observed were ST307/Tn4401a[blaKPC-2], ST307/Tn4401b[blaKPC-4], ST11/Tn4401a[blaKPC-2], and Tn4401b[blaKPC-2], while the observed $E$. coli clones were ST410/Tn4401a [blaKPC-2] and ST3520/Tn4401a[blaKPC-2]. Importantly, the Tn4401 isotypes differed in the promoter of the blaKPC gene, which was closely associated with the level of gene expression and caused variation in carbapenem susceptibility [12]; however, there was no significant difference in susceptibility in this study.

$K$. pneumoniae ST307 and E. coli ST410 have garnered attention as potentially high-risk CPE producers and are associated with a notorious clone, CTX-M-15 [44]. Furthermore, clones co-producing CPE and CTXM-15 were recently identified in Italy [45] and Denmark [46]. The high distribution of CTX-M-1 reported in this study highlights the risk of emergence and spread of high-risk clones (Table 1). High-risk clones are globally distributed and associated with various antimicrobial resistance determinants, such as ease of transmission, persistence in hosts, and effective transmission between hosts. These high-risk clones possess enhanced pathogenicity and are more prone to cause severe and recurrent infections. Thus, considering the clonal expansion over the past decades and increased antimicrobial resistance, ST307 and ST410 should be prospectively monitored in South Korea.

The CPE incidence and molecular genetic traits observed at this hospital revealed trends similar to those reported in the Disease Control Headquarters survey conducted in 2017 [47]. However, the CPE incidence rate increased by $25 \%$ and that of CRE increased by $5 \%$ in 2018 relative to those in 2017 . While the previous CRE occurrence in Korea was characterized by ESBL, AmpC, or porin loss, the current study reveals that carbapenemase-producing bacteria currently represent the greatest risk to public health [31]. Transfer by conjugation accounted for $57.5 \%$ of resistant isolates, which is 4.3 fold higher than that found in the 2010 study [14]. Furthermore, the high detection rate found for transposon Tn4401 revealed the rapid spread and evolution of CPE.

In summary, our study highlights that carbapenem resistance can be transferred among strains through transconjugation processes, which results in a higher number of strains with reduced carbapenem susceptibility. We found that the causes of carbapenem resistance spread were plasmids and transposons; therefore, more efforts are needed to control the spread of CPE in hospitals. The treatment of CRE infections often involves a combination therapy, including carbapenem, aminoglycosides, and fosfomycin; however, this therapeutic option is not yet available in South Korea. Thus, medical institutions should monitor whether carbapenem-resistant enterobacteria-infected patients are isolated, and if so, these institutions should prevent the spread through infection control measures, such as patient contact tracing, thorough use of personal protective equipment, and contact inspection. Systematic monitoring and regulation alone will enable better control and prevent the spread of KPC producers.

\section{Acknowledgment}

This research was supported by the BHS Hanseo Hospital in 2019.

\section{Conflict of Interest}

The authors have no financial conflicts of interest to declare.

\section{References}

1. O'Neill J. 2014. Antimicrobial resistance: Tackling a crisis for the health and wealth of nations. Review of antimicrobial resistance. Available from https://amr-review.org/sites/default/files/AMR\%20 Review\%20Paper\%20-\%20Tackling\%20a\%20crisis\%20for\%20the \%20health\%20and\%20wealth\%20of\%20nations_1.pdf. Accessed 
Nov. 20, 2019.

2. Centers for Disease Control and Prevention. 2013. Antibiotic resistance threats in the United States, 2013. Available from https:// www.cdc.gov/drugresistance/pdf/ar-threats-2013-508.pdf. Accessed Nov. 17, 2019.

3. Kim JO, Song SA, Yoon EJ, Shin JH, Lee H, Jeong SH, et al. 2017. Outbreak of KPC-2-producing Enterobacteriaceae caused by clonal dissemination of Klebsiella pneumoniae ST307 carrying an IncX3-type plasmid harboring a truncated Tn4401a. Diagn. Microbiol. Infect. Dis. 87: 343-348.

4. Jeong SH, Kim HS, Kim JS, Shin DH, Kim HS, Park MJ, et al. 2016. Prevalence and molecular characteristics of carbapenemaseproducing Enterobacteriaceae from five hospitals in Korea. Ann. Lab. Med. 36: 529-535.

5. Mouloudi E, Protonotariou E, Zagorianou A, losifidis E, Karapanagiotou A, Giasnetsova T, et al. 2010. Bloodstream infections caused by metallo- $\beta$-lactamase/Klebsiella pneumoniae carbapenemase-producing $K$. pneumoniae among intensive care unit patients in Greece: Risk factors for infection and impact of type of resistance on outcomes. Infect. Control Hosp. Epidemiol. 31: 12501256.

6. Bradford PA, Urban C, Mariano N, Projan SJ, Rahal JJ, Bush K. 1997. Imipenem resistance in Klebsiella pneumoniae is associated with the combination of ACT-1, a plasmid-mediated AmpC $\beta$-lactamase, and the foss of an outer membrane protein. Antimicrob. Agents Chemother. 41: 563-569.

7. Kaczmarek FM, Dib-Hajj F, Shang W, Gootz TD. 2006. High-level carbapenem resistance in a Klebsiella pneumoniae clinical isolate is due to the combination of bla $a_{\text {ACT-1 }} \beta$-lactamase production, porin OmpK35/36 insertional inactivation, and down-regulation of the phosphate transport porin PhoE. Antimicrob. Agents Chemother. 50: 3396-3406.

8. Nordmann P, Dortet L, Poirel L. 2012. Carbapenem resistance in Enterobacteriaceae: here is the storm! Trends Mol. Med. 18: 263272.

9. Giani T, Pini B, Arena F, Conte V, Bracco S, Migliavacca R, et al. 2013. Epidemic diffusion of KPC carbapenemase-producing Klebsiella pneumoniae in Italy: Results of the first countrywide survey, 15 May to 30 June 2011. Euro. Surveill. 18: 20489.

10. Lee CR, Lee JH, Park KS, Kim YB, Jeong BC, Lee SH. 2016. Global dissemination of carbapenemase-producing Klebsiella pneumoniae: Epidemiology, genetic context, treatment options, and detection methods. Front. Microbiol. 7: 895.

11. Chen YT, Lin JC, Fung CP, Lu PL, Chuang YC, Wu TL, et al. 2014. KPC-2-encoding plasmids from Escherichia coli and Klebsiella pneumoniae in Taiwan. J. Antimicrob. Chemother. 69: 628-631.

12. Frost L, Leplae R, Summers AO, Toussaint A. 2005. Mobile genetic elements: The agents of open source evolution. Nat. Rev. Microbiol. 3: 722-732.

13. Sota M, Top E. 2008. Horizontal gene transfer mediated by plasmids, pp. 111-181. In Lipps G (ed.), Plasmids: Current Research and Future Trends. Caister Academic Press, Horizon Scientific Press, Norfolk, VA.
14. Frost LS, Koraimann G. 2010. Regulation of bacterial conjugation: Balancing opportunity with adversity. Future Microbiol. 5: 10571071.

15. Smillie C, Garcillán-Barcia MP, Francia MV, Rocha EP, de la Cruz F. 2010. Mobility of plasmids. Microbiol. Mol. Biol. Rev. 74: 434-452.

16. Guglielmini J, Quintais L, Garcillán-Barcia MP, de la Cruz F, Rocha EP. 2011. The repertoire of ICE in prokaryotes underscores the unity, diversity, and ubiquity of conjugation. PLoS Genet. 7: e1002222.

17. Naas T, Cuzon G, Truong HV, Nordmann P. 2012. Role of ISKpn7 and deletions in bla $a_{\mathrm{KPC}}$ gene expression. Antimicrob. Agents Chemother. 56: 4753-4759.

18. Cicek AC, Duzgun AO, Saral A, Sandalli C. 2014. Determination of a novel integron-located variant (bla $\left.a_{\mathrm{OXA}-320}\right)$ of Class $D \beta$-lactamase in Proteus mirabilis. J. Basic Microbiol. 54: 1030-1035.

19. Clinical and Laboratory Standards Institute. 2018. Methods for dilution antimicrobial susceptibility tests for bacteria that grow aerobically. Wayne, Pennsylvania.

20. Jeong S, Kim JO, Jeong SH, Bae IK, Song W. 2015. Evaluation of peptide nucleic acid-mediated multiplex real-time PCR kits for rapid detection of carbapenemase genes in gram-negative clinical isolates. J. Microbiol. Methods. 113: 4-9.

21. Pérez-Pérez FJ, Hanson ND. 2002. Detection of plasmid-mediated AmpC $\beta$-lactamase genes in clinical isolates by using multiplex PCR. J. Clin. Microbiol. 40: 2153-2162.

22. Ryoo NH, Kim EC, Hong SG, Park YJ, Lee K, Bae IK, et al. 2005. Dissemination of SHV-12 and CTX-M-type extended-spectrum $\beta$ lactamases among clinical isolates of Escherichia coli and Klebsiella pneumoniae and emergence of GES-3 in Korea. J. Antimicrob. Chemother. 56: 698-702.

23. Yamane K, Wachino J, Suzuki S, Arakawa Y. 2008. Plasmid-mediated qepA gene among Escherichia coli clinical isolates from Japan. Antimicrob. Agents Chemother. 52: 1564-1566.

24. Landman D, Bratu S, Quale J. 2009. Contribution of OmpK36 to carbapenem susceptibility in KPC-producing Klebsiella pneumoniae. J. Med. Microbiol. 58: 1303-1308.

25. Yoon E-J, Kim JO, Kim D, Lee H, Yang JW, Lee KJ, et al. 2018. Klebsiella pneumonia carbapenemase producers in South Korea between 2013 and 2015. Front. Microbiol. 9:56.

26. Wirth T, Falush D, Lan R, Colles F, Mensa P, Wieler LH, et al. 2006. Sex and virulence in Escherichia coli: an evolutionary perspective. Mol. Microbiol. 60: 1136-1151.

27. Diancourt L, Passet V, Verhoef J, Grimont PA, Brisse S. 2005. Multilocus sequence typing of Klebsiella pneumoniae nosocomial isolates. J. Clin. Microbiol. 43: 4178-4182.

28. Jeong SH, Lee KM, Lee J, Bae IK, Kim JS, Kim HS, et al. 2015. Clonal and horizontal spread of the bla ${ }_{\mathrm{OXA}-232}$ gene among Enterobacteriaceae in a Korean hospital. Diagn. Microbiol. Infect. Dis. 82: 70-72.

29. Leavitt A, Chmelnitsky I, Ofek I, Carmeli Y, Navon-Venezia S. 2010. Plasmid pKpQIL encoding KPC-3 and TEM-1 confers carbapenem resistance in an extremely drug-resistant epidemic Klebsiella pneumoniae strain. J. Antimicrob. Chemother. 65: 243-248.

30. Tsai YK, Fung CP, Lin JC, Chen JH, Chang FY, Chen TL, et al. 2011. 
Klebsiella pneumoniae outer membrane porins OmpK35 and OmpK36 play roles in both antimicrobial resistance and virulence. Antimicrob. Agents Chemother. 55: 1485-1493.

31. Kim SY, Shin J, Shin SY, Ko KS. 2013. Characteristics of carbapenem-resistant Enterobacteriaceae isolates from Korea. Diagn. Microbiol. Infect. Dis. 76: 486-490.

32. Schulz J, Kemper N, Hartung J, Janusch F, Mohring SAl, Hamscher G. 2019. Analysis of fluoroquinolones in dusts from intensive livestock farming and the co-occurrence of fluoroquinolone-resistant Escherichia coli. Sci. Rep. 9:5117.

33. Dalhoff A. 2012. Global fluoroquinolone resistance epidemiology and implications for clinical use. Interdiscip. Perspect. Infect. Dis. 2012: 976273.

34. Sárközy G. 2001. Quinolones: a class of antimicrobial agents. Vet. Med. 46: 257-274.

35. Grobbel M, Lübke-Becker A, Wieler LH, Froyman R, Friederichs S, Filios S. 2007. Comparative quantification of the in vitro activity of veterinary fluoroquinolones. Vet. Microbiol. 124: 73-81.

36. Frye JG, Jackson CR. 2013. Genetic mechanisms of antimicrobial resistance identified in Salmonella enterica, Escherichia coli, and Enteroccocus spp. isolated from U.S. food animals. Front. Microbiol. 4: 135.

37. Hopkins KL, Davies RH, Threlfall EJ. 2005. Mechanisms of quinolone resistance in Escherichia coli and Salmonella: Recent developments. Int. J. Antimicrob. Agents 25: 358-373.

38. Endtz HP, Ruijs GJ, van Klingeren $B$, Jansen WH, van der Reyden $T$, Mouton RP. 1991. Quinolone resistance in campylobacter isolated from man and poultry following the introduction of fluoroquinolones in veterinary medicine. J. Antimicrob. Chemother. 27: 199-208.

39. García Ovando H, Gorla N, Luders C, Poloni G, Errecalde C, Prieto $\mathrm{G}$, et al. 1999. Comparative pharmacokinetics of enrofloxacin and ciprofloxacin in chickens. J. Vet. Pharmacol. Ther. 22: 209-212.

40. van den Bogaard AE, London N, Driessen C, Stobberingh EE. 2001. Antibiotic resistance of faecal Escherichia coli in poultry, poultry farmers and poultry slaughterers. J. Antimicrob. Chemother. 47: 763-771.

41. Leclercq R, Cantón R, Brown DF, Giske CG, Heisig P, MacGowan AP, et al. 2013. EUCAST expert rules in antimicrobial susceptibility testing. Clin. Microbiol. Infect. 19: 141-160.

42. Sheppard AE, Stoesser N, Wilson DJ, Sebra R, Kasarskis A, Anson LW, et al. 2016. Nested Russian doll-like genetic mobility drives rapid dissemination of the carbapenem resistance gene $b / a_{\mathrm{KPC}}$. Antimicrob. Agents Chemother. 60: 3767-3778.

43. Pitout JD, Nordmann P, Poirel L. 2015. Carbapenemase-producing Klebsiella pneumoniae, a key pathogen set for global nosocomial dominance. Antimicrob. Agents Chemother. 59: 5873-5884.

44. Villa L, Feudi C, Fortini D, Brisse S, Passet V, Bonura C, et al. 2017. Diversity, virulence, and antimicrobial resistance of the KPC-producing Klebsiella pneumoniae ST307 clone. Microb. Genom. 3: e000110.

45. Geraci DM, Bonura C, Giuffrè M, Saporito L, Graziano G, Aleo A, et al. 2015. Is the monoclonal spread of the ST258, KPC-3-producing clone being replaced in southern Italy by the dissemination of multiple clones of carbapenem-nonsusceptible, KPC-3-producing Klebsiella pneumoniae? Clin. Microbiol. Infect. 21: e15-e17.

46. Roer L, Overballe-Petersen S, Hansen F, Schønning K, Wang M, Røder BL, et al. 2018. Escherichia coli sequence type 410 is causing new international high-risk clones. mSphere 3: e00337-18.

47. Korea Centers for Disease Control and Prevention. Distribution of carbapenem-resistant Enterobacteriaceae (CRE) in Korea, 2017. Available from https://is.cdc.go.kr/upload_comm/syview/doc. html?fn=156811210482900.pdf\&rs=/upload_comm/docu/0034/. Accessed Dec. 11, 2019. 